\title{
La inclusividad lingüística en la educación multilingüe de California: coexistencia de las variedades y registros de lengua para enriquecer el aula de inmersión dual
}

\section{Jordi Solsona-Puig}

orcid.org/0ooo-0001-5522-3189

Pepperdine University, Estados Unidos

de América.

jordi.solsona-puig@pepperdine.edu
María Capdevila-Gutiérrez

orcid.org/000o-0003-0131-3247

Glendale Unified School District

California, Estados Unidos de América

mcapdevila@gusd.net

\section{Fernando Rodríguez-Valls}

orcid.org/oooo-ooo1-6564-6863

California State University, Fullerton,

Estados Unidos de América

frodriguez-valls@fullerton.edu

\section{Resumen}

En las aulas de inmersión dual de California, múltiples variedades, niveles y registros lingüisticos conviven cuando los estudiantes y maestros nativos en la lengua de instrucción aportan la riqueza de sus diferentes origenes lingüisticos. Es sabido que el español es el idioma con mayor presencia dentro de estos programas en todo Estados Unidos. No obstante, el dilema de cómo enfrentarse a la coexistencia de diferentes variedades de un mismo idioma de instrucción afecta también a programas de inmersión de forma transnacional. Consecuentemente, otras tantas modalidades pueden llegar a convivir en el aula de inmersión dual, en especial cuando los estudiantes hispanohablantes tienen raíces inmigrantes de múltiples orígenes geográficos y socioeconómicos. Lejos de la aceptación exclusiva de una modalidad única o preferible, en este artículo se propone que el aula de inmersión dual sea un ejemplo de inclusividad y respeto por las múltiples variedades lingüísticas como fuente de riqueza trasladable al aprendizaje de cualquier idioma. Un aula lingüisticamente inclusiva se antoja más respetuosa con las múltiples identidades de sus hablantes $y$ acorde con la naturaleza viva de cualquier idioma, especialmente en sociedades multilingües. Finalmente, el artículo incluye recomendaciones tanto para la instrucción de lengua y contenidos como para la capacitación de maestros bilingües desde la perspectiva de inclusividad lingüistica.

\section{Palabras clave}

California - EE.UU., educación multilingüe, enseñanza de idiomas, formación de profesores, inclusión lingüística (Fuente: Tesauro de la Unesco).

Recepción: 13/01/2018 | Envío a pares: 03/04/2018 | Aceptación por pares: 16/04/2018 | Aprobación: 24/06/2018

DOI: 10.5294/edu.2018.21.2.3

Para citar este artículo / To reference this article / Para citar este artigo

Solsona-Puig, J., Capdevila-Gutiérrez, M. y Rodríguez-Valls, F. (2018). La inclusividad lingüística en la educación multilingüe de California: coexistencia de las variedades y registros de lengua para enriquecer el aula de inmersión dual. Educación y Educadores, 21(2), 219-236. doi: 10.5294/edu.2018.21.2.3 


\title{
Linguistic Inclusiveness in Multilingual Edu- cation in California: Coexistence between Language Varieties and Registers to Enrich Dual Immersion Classrooms
}

\begin{abstract}
In California's dual immersion classrooms, multiple varieties, levels and linguistic registers coexist when students and teachers whose mother tongue is the language of instruction contribute the richness of their different linguistic origins. Spanish is known to be the predominant language within these programs throughout the United States. However, the dilemma of how to deal with the coexistence of different varieties of the same language of instruction also affects immersion programs in other languages. Consequently, other modalities can coexist in a dual immersion classroom, especially when Spanish-speaking students have immigrant roots of various geographical and socio-economic origins. Far from accepting a single or preferable arrangement exclusively, this article proposes the dual immersion classroom be an example of inclusiveness and respect for the diversity of multiple linguistic varieties as a source of transferable wealth for learning any language. A linguistically inclusive classroom seems more respectful of the multiple identities of its speakers and in keeping with the living nature of any language, especially in multilingual societies. The article ends with recommendations for language and content instruction as well as for training bilingual teachers from the standpoint of linguistic inclusivity.
\end{abstract}

\section{Keywords}

California, USA, multilingual education, language of instruction, teacher training, linguistic inclusion (Source: UNESCO Thesaurus). 


\section{A inclusão linguística na educação multilín- gue da Califórnia: coexistência de variedades e registros de língua para enriquecer a sala de aula de imersão dual}

Resumo

Nas salas de aula de imersão dual da Califórnia, múltiplas variedades, níveis e registros linguísticos coexistem quando os estudantes e os professores nativos da língua de instrução contribuem para a riqueza de suas diferentes origens linguísticas. Sabe-se que o espanhol é a língua com maior presença dentro desses programas nos Estados Unidos. No entanto, o dilema de como trabalhar com a coexistência de diferentes variedades de um mesmo idioma de instrução afeta também os programas de imersão em outras línguas. Como consequência, muitas outras modalidades podem chegar a conviver na sala de aula de imersão dual, especialmente quando os estudantes hispanofalantes têm raízes imigrantes de múltiplas origens geográficase socioeconômicas. Longe da aceitação exclusiva de uma modalidade única ou preferivel, este artigo propõe que a sala de aula de imersão dual deve ser um exemplo de inclusão e respeito à diversidade de múltiplas variedades linguísticas como fonte de riqueza transferivel à aprendizagem de qualquer idioma. Uma sala de aula linguisticamente inclusiva parece respeitar mais as múltiplas identidades de seus falantes e concorda com a natureza viva de qualquer idioma, especialmente em sociedades multilingues. Finalmente, o artigo inclui recomendações tanto para a instrução de língua e seus conteúdos como para a formação de professores bilingues a partir da perspectiva da inclusão linguística.

\section{Palavras-chave}

Educação multilíngue, Inclusão linguistica, ensino de idiomas; formação de professores; Califórnia - E.U.A. (Fonte: Tesauro da Unesco). 
El conocimiento de las actitudes lingüisticas de los hablantes de una comunidad resulta fundamental para comprender cómo mediante el lenguaje se configuran y se difunden ideologías, representaciones sociales e identidades, sean estas individuales o grupales. (Rojas, 2014, p. 251)

Los programas de inmersión dual, conocidos en lengua inglesa como Dual Immersion o Two-Way Immersion (TWI), cuentan con una tradición de más de sesenta años en Estados Unidos. Inicialmente diseñados para mejorar los niveles de literacidad y el rendimiento académico de los estudiantes de grupos minoritarios hablantes de una lengua distinta al inglés (Alfonso, 2017), pronto se propagaron (CAL, 2018a) debido al interés creciente de las familias hablantes de inglés como primer idioma, a quienes atrajo la perspectiva de una educación bilingüe enriquecedora, de alta calidad y de comprobado éxito académico (Lindholm-Leary y Hernández, 2011; Marian, Shook y Schroeder, 2013). En los últimos veinte años, y a pesar de la existencia de una legislación diseñada para favorecer un sistema educativo monolingüe, los partidarios de la diversidad lingüística en California han trabajado para ver cumplida la promesa de una educación bilingüe de calidad (Lindholm-Leary, 2005; Palmer, 2007). Una vez aprobada la Proposición $58^{1}$ (CLI, 2018), se sientan las bases de un nuevo marco legislativo más favorable a un número creciente de programas educativos que tienen como objetivo la biliteracidad desde una perspectiva multilingüe inclusiva.

A pesar de la existencia de múltiples tipologías de programas inmersión dual, estos deben cumplir tres características para ser considerados de calidad: 1) la separación de las dos lenguas de instrucción: lengua meta e inglés; 2) un mínimo del 50\% de instrucción en la lengua meta; 3 ) un compromiso de permanencia desde hasta el final de la escuela secundaria (De Jong, 2016) y, en cualquier caso, una permanencia mínima de seis años (Lindholm-Leary, 2005). Otros as-

1 La Proposición 58 (2016) revocó en California parte de la regulación restrictiva establecida en la Proposición 227 (1997) que limitaba solamente al inglés la instrucción de los estudiantes aprendices de este idioma. pectos que determinan la calidad de los programas son: un número equilibrado de hablantes nativos de inglés y de la lengua meta; una aproximación a la inmersión dual, entendida como una educación enriquecedora y no remedial, y una instrucción tanto para la literacidad como en los contenidos curriculares en ambas lenguas (De Jong y Howard, 2009).

Por lo que respecta al tiempo de instrucción, hay dos sistemas claramente diferenciados (CAL, 2018b). En el modelo denominado 50:50, la mitad de la instrucción se imparte en inglés y la otra mitad en la lengua meta. Existe otro modelo de inclusión gradual del inglés en la instrucción frecuentemente llamado 90:10. En este modelo, los estudiantes comienzan utilizando la lengua meta un noventa por cien de la instrucción. A medida que se avanza en el nivel educativo, el porcentaje de la lengua minoritaria disminuye y el porcentaje en inglés se incrementa, hasta llegar a 50:50 al final de la educación primaria. Este modelo gradual, que se ha mostrado como el más efectivo (Potowski, 2004; CDE, 2018b), se utiliza para proteger la lengua minoritaria del dominio de la lengua dominante: el inglés (Palmer, 2007). Sea cual sea el modelo de inmersión, el objetivo común es crear un estudiante con altos niveles de biliteracidad.

Para asegurar que estos niveles de biliteracidad y éxito académico se cumplan, el presente artículo propone la consideración de nuevos aspectos, tales como: el rol inclusivo de la lengua minoritaria en los procesos de instrucción, que en este caso es abrumadoramente el español; las múltiples variedades y registros de la lengua meta; y el modo en que estas prácticas inclusivas afectan a las actitudes lingüísticas e identitarias de los hablantes en el aula. Por último, indagamos en la repercusión de estas prácticas inclusivas en la formación de los maestros bilingües. Dichas consideraciones pueden extrapolarse a cualquier programa de inmersión, independientemente 
de la lengua de instrucción, pero la apuesta por la educación multilingüe en California nos brinda una oportunidad única de análisis.

La constante evolución que afecta a cualquier lengua viva se ve amplificada en el estado de California debido a una multiplicidad de factores geográficos, socioeconómicos, histórico-políticos y lingüísticos. No en vano, según el análisis de los datos del U.S. Census Bureau (USCB, 2018a), California es, en muchos sentidos, el territorio más diverso² de los Estados Unidos de América. No es casualidad que EE.UU. sea, ahora mismo, el segundo país del mundo en número de hablantes nativos de español, con 39,1 millones, justo después de México. Solo en California, la lengua de Borges y García Márquez es el idioma nativo de más de una cuarta parte de la población, con un número aproximado de 10,4 millones de hispanohablantes (USCB, 2018b) y un tercio de los alumnos en edad escolar (CDE, 2018a). La interacción entre el inglés y el español es visible en casi todos los ámbitos de la vida cotidiana. No obstante, este contacto entre lenguas se reduce y limita cuando se trata de los ámbitos institucional y educativo, en los que el inglés es claramente dominante. Como explica Altbach, "el inglés ejerce ahora mismo, de manera incuestionable, como el principal idioma académico internacional. De hecho, los sistemas académicos nacionales dan la bienvenida al inglés como lengua que contribuye a su internacionalización, competitividad y a alcanzar el estatus de primera categoría mundial"3 (2007, p. 2).

2 Aplicando la fórmula Herfindahl-Hirschman a los datos de población del US Census Bureau (2017) para hallar el índice de diversidad a un conjunto de aspectos como el origen étnico, nivel educativo, salud, nivel ocupacional, economía y nivel de ingresos/pobreza, el estado californiano resulta ser el más diverso de EE.UU.

3 En adelante, todas las citas en inglés han sido traducidas por los autores. En el original: "English now serves unchallenged as the main international academic language. Indeed, national academic systems enthusiastically welcome English as a contributor to internationalizing, competing, and becoming 'world class'".
En California, al igual que en otros estados del sur de Estados Unidos, como Texas, Florida o Arizona, donde confluyen un gran número de variedades y registros de la lengua española, la principal puerta de entrada de este idioma es la frontera. Esta frontera geopolítica no impide que el español cruce a través de ella sin necesidad de ningún tipo de documentación. La frontera, descrita por Anzaldúa como una herida sangrante de personas (2012), sangra también inevitablemente cultura y, con esta, idiomas. Ada y Zubizarreta explican por medio de la narración que quien cruza la frontera siempre lleva consigo la lengua, la cual no requiere documentos ni permisos para pasar: "aludiendo a que algunas personas llaman despectivamente espaldas mojadas a quienes cruzan la frontera sin documentos, todo el día preguntaba: '¿Ya se le secó la espalda a tu prima?”, ‘Ya sabe hablar?’ o ‘Se le quedó la lengua en México?"' (2013, p. 26). El español permea desde México hacia el norte con la gran riqueza y variedad lingüística que traen emigrantes de América Latina y los medios de comunicación que sirven al gran número de hablantes latinos residentes en estos estados (Alim, 2005; Showstack, 2012; Neroy Ahmad, 2014). De manera similar, las numerosas variedades del español llegan al aula de la mano de estudiantes y maestros, que usan, comparten y transforman una lengua rica y viva que aspira a convivir de tú a tú con el inglés.

Dentro de esta corriente bidireccional es donde los estudiantes hispano-parlantes desarrollan y forman su identidad lingüistica y cultural. Una identidad que por momentos se siente monolingüe, ya sea en español o inglés, o que se alimenta de ambas lenguas. Anzaldúa dibuja esta ambigüedad describiendo la identidad étnica en estrecha y necesaria relación con la identidad lingüística. Para la autora, "la identidad étnica es gemela de la identidad lingüística. Yo soy mi lengua. Hasta que no me sienta orgullosa de mi lengua, no puedo sentirme orgullosa de mí misma... Hasta que no me sienta libre de escribir de 
una manera bilingüe y poder cambiar de código sin tener que traducir"4 (2012, p. 81).

Este artículo explora cómo el español y sus distintas variedades pueden y deben ser utilizados en el aula de inmersión dual, considerada como paradigma de una educación de calidad. Partiendo de la modalidad estándar de la lengua de instrucción, proponemos que, en la interacción en el aula, cada hablante utilice la variedad que lo defina natural y lingüísticamente desde un punto de vista inclusivo (Ibarra, 2012; Rodríguez, Carrasquillo y Soon, 2014). Únicamente así se conseguirá convertir la lengua de instrucción en la herramienta de unión que debe ser en este tipo de aula. A pesar de la absoluta necesidad de rigor lingüístico, nos parece necesaria la celebración e inclusión de las muchas diferencias asociadas a esta lengua. De igual manera, pensamos que en el aula se deben explorar cuestiones referentes a la constante evolución del lenguaje gracias a su riqueza dialectal y de registros o a la simple influencia mutua de idiomas en contacto. A efectos de este estudio, utilizaremos el concepto de español estándar como el modelo de lengua generalizado, desde un punto de vista sociolingüístico. Su definición corresponde a la "variante de prestigio usada por una comunidad de habla, que trasciende las diferencias geográficas y provee una modalidad unificada que puede ser usada por los medios de comunicación y por la escuela" (Alba, 2001, p. 2). En la misma fuente, el autor advierte de las connotaciones peyorativas y la falta de prestigio de las llamadas variedades no estándar o subestándar, usadas en zonas rurales y por los sectores socioeconómicos bajos de las ciudades.

\section{La clase de español: celebración de las variedades de la lengua}

Los factores económicos, sociales y políticos que determinan cualquier idioma influyen de for-

4 En el original: "Ethnic identity is twin skin to linguistic identity— I am my language. Until I take pride in my language, I cannot take pride in myself ... Until I am free to write bilingually and to switch codes without having to translate". ma ostensible tanto en el uso como en la enseñanza del idioma en cuestión (Lomas, 2014). Curiosamente, el mismo idioma acarrea connotaciones totalmente diferentes según el grupo y los objetivos de las personas que lo están utilizando o aprendiendo. En este sentido, enseñar español a hablantes de otra lengua se percibe como una fuente de posibilidades de crecimiento personal, éxito académico y mejora profesional y económica (Martín, 2010). En otros grupos, sin embargo, incluso dentro de una misma aula, el aprendizaje del idioma se percibe como una cuestión remedial y necesaria para suplir deficiencias lingüísticas y, en ocasiones, académicas (RodríguezValls, Solsona-Puig y Capdevila-Gutiérrez, 2017). Desde este punto de partida, el maestro de programas de inmersión dual debe luchar activamente contra preconcepciones con las que a menudo los propios estudiantes, sus familias o el mismo docente llegan al aula (Gándara, 2010; Shin, 2012). En el caso particular del español, lengua hablada en más de una veintena de países, estas preconcepciones y prejuicios se multiplican debido a las diferentes connotaciones nacionales, políticas o económicas que las distintas variedades nacionales de esta lengua traen consigo. Tal y como apunta el informe El español, una lengua viva, la importancia de una lengua, o de sus distintos dialectos, suele medirse teniendo en cuenta factores económicos muy diversos, como "su número de hablantes, su extensión geográfica, el número de países en los que tiene rango de oficial, el índice de desarrollo humano de sus hablantes" (Fernández, 2017, p. 27). En el docente del aula de español en inmersión dual recae una tarea doble. Por un lado, debe centrarse en la enseñanza de la lengua, la literatura y la cultura asociadas a esta y, por otro, debe trabajar consistentemente para derribar esas concepciones erróneas y dañinas que afectan no solo a la lengua en sí, sino al sentimiento identitario de sus hablantes. Este trabajo bidireccional del docente afectará, por ende, la sensación de pertenencia al entorno educativo del alumno y, con todo ello, a su rendimiento académico (García y Kleifgen, 2010; Lindholm-Leary y Hernández, 2011 y Rodríguez-Valls y Capdevila-Gutiérrez, 2017). 
En los programas de inmersión dual, aproximadamente la mitad de los estudiantes, además del maestro, traen al aula distintas variedades dialectales de español. La otra mitad, hablantes nativos de otros idiomas, aportan una variedad de español como segundo idioma, la cual se ve influenciada a su vez por los distintos registros del conjunto de compañeros y maestros que ha tenido a lo largo del programa. La variedad de orígenes nacionales de maestros y alumnos se ve reflejada también en el idioma del aula en cuestiones léxicas, gramaticales y fonético-fonológicas (Hornberger, 2009). Todas estas cuestiones deben tratarse de forma explícita en el aula, ya que ofrecen una enriquecedora excusa para la discusión metalingüística y el conocimiento profundo de la lengua meta. El acceso a la diversidad supone un motivo de aprendizaje y ensalzamiento de las distintas culturas representadas en el aula. Más allá del ámbito del lenguaje oral, los distintos orígenes y variedades lingüísticas de los miembros del aula de inmersión dual deben verse apoyados por un estudio inclusivo y variado de textos literarios, históricos, periodísticos, etc., de los países y dialectos representados en el aula, incluyendo entre estos la lengua y cultura en español en los Estados Unidos. Es importante, tal y como sugiere Cassany, que los maestros diseñen tareas con varios textos - en este caso, con registros diferentes- "con documentos múltiples o paralelos [...]. Todo ello facilita que entren en el aula puntos de vista diferentes e incluso contradictorios" (2010, p. 36 ).

Como hemos explicado anteriormente, a partir de una variedad estándar compartida por todos los miembros del aula, se debe trabajar en dos direcciones: hacia una evolución del español correcto y académico, así como hacia un mayor conocimiento y puesta en valor de las distintas variedades dialectales de esta lengua. Partiendo de las similitudes, las diferencias, lejos de ser un obstáculo, son una herramienta de debate y aprendizaje lingüístico y cultural. Entender las variedades léxicas o gramaticales como alternativas enriquecedoras y sus referentes y conexiones culturales como elementos propios de la lengua y culturas asociadas a esta favorece la puesta en valor de las culturas de origen de todos los participantes del aula (Trujillo, Lorenzo y Vez, 2011).

Tratándose de programas de inmersión dual en California es necesario hacer una referencia al uso del español con influencias del inglés. Dado que hemos enfatizado sobre la necesidad de considerar todas las variedades dialectales como opciones igualmente apropiadas y al respecto de la mencionada corrección lingüística y el lenguaje académico, nos parece necesario hacer una reflexión sobre el mestizaje lingüístico. El docente, tratando de potenciar la corrección lingüística y el español académico, incurre a menudo en comentarios y explicaciones que conllevan valoraciones y juicios sobre el uso que los estudiantes hacen de la lengua. Si bien es importante que los estudiantes adquieran las herramientas para discernir lo que, dentro del lenguaje estándar, se considera lenguaje correcto y lo que no, es necesario también entender que esa variedad de uso es, no solo la elegida por los estudiantes, sino la que representa su identidad cultural. El maestro del aula de inmersión dual en California y, por extensión, en el resto de los Estados Unidos debe tener presente que muchos de los estudiantes de programas de inmersión dual se sienten individuos "mestizos", no solo por su origen, sino por su cultura y lengua. De este modo, su variedad lingüística familiar o cultural no pertenece a un registro nacional único, sino a varios o a una combinación de registros y lenguas. Esto es lo que consideramos mestizaje lingüístico y que Betancur García explica de la siguiente manera:

En el mestizaje, los individuos y los grupos que son fruto de dichas mixturas sintetizan y ensamblan caracteristicas de las culturas precedentes. Por tanto, los individuos que nacen son nuevos y distintos. Los elementos que más fuertemente permiten el mestizaje o el tejido cultural son la lengua, la religión, el arte, la comida, el folklore y la educación, especialmente si las 
culturas encontradas han tenido algún nivel de apertura entre si. (2014, p. 106)

A menudo, muchos de estos estudiantes se encuentran a caballo entre dos o más nacionalidades, culturas e idiomas (Paffey, 2014). Por ello, y porque en la representación de su identidad mestiza eligen una forma específica de lenguaje (léxico, acento, etc.), sería altamente contraproducente marcar esa variedad como errónea o inferior. El refuerzo positivo, por medio de la puesta en valor de los distintos registros, ayuda a que el joven hablante sea capaz de entender y elegir. Es necesario que los alumnos entiendan que palabras como "troca" o "parquear" o expresiones como "venir o llamar para atrás" se consideran erróneas en español (Rodríguez-Valls, 2009). No obstante, el maestro debe entender que esas expresiones y palabras son un registro vivo que describe la realidad y el contexto cultural y social de muchos de estos estudiantes. De este modo, el maestro les otorga el conocimiento lingüístico y la posibilidad de elegir el uso de la lengua y el registro de ella en función de sus necesidades y sus intereses (Livingstone y Ferreira, 2009; Montrul, 2012). El alumno bilingüe tiene así la posibilidad de cambiar, no solo de código lingüístico, sino de registro según su conveniencia. Esta visión inclusiva de la lengua de instrucción hace que el español, en el aula de inmersión dual, sea una herramienta para la comunicación y la puesta en valor de las culturas, sea cual fuere la variedad o dialecto de lengua que maestros y estudiantes utilicen.

\section{Orientaciones para el uso inclusivo de la lengua de instrucción en el aprendizaje integrado de contenidos}

Frecuentemente, en la literatura sobre educación bilingüe, los conceptos de inmersión y aprendizaje integrado de contenidos (CLIL) se utilizan indistintamente. A pesar de ello, algunos autores defienden que, aunque el CLIL deriva de los métodos de instrucción de una segunda lengua por inmersión, actualmente las diferencias entre estos dos conceptos superan a las similitudes (Lasagabaster y Sierra, 2009). Independientemente de este matiz, en los programas de inmersión dual el aprendizaje integrado de contenidos debe fomentar la lengua de instrucción como el vehículo para la equidad pedagógica, en tres vertientes: la rigurosidad lingüística, la exhaustividad del contenido y la inclusividad de la diversidad de registros y variedades lingüísticas. Este último aspecto, la inclusividad lingüística como parte de una educación multicultural (Ghosh y Galczynski, 2014), será el foco de este apartado.

Es sabido que la instrucción rigurosa de contenidos lingüísticos, o sea, un currículum enriquecido o de calidad (Howard, Lindholm-Leary, Sugarman, Christian y Rogers, 2007), ha sido una de las garantías de éxito a la vez que una seña de identidad de los programas de inmersión. Al mismo tiempo, se han mantenido altos estándares de calidad en los contenidos para alumnos hispanohablantes. Así, Alanís y Rodríguez exponen que "el éxito académico y lingüístico de niños en inmersión dual que tienen el español como lengua dominante puede ser atribuido al uso de currículo general que se enseña en la lengua nativa del niño, así como a los rigurosos estándares de contenido, independientemente del lenguaje de instrucción"5 (2008, p. 302). La búsqueda de la instrucción con armonía entre los aspectos lingüísticos y los contenidos debe ser complementada con el tercer elemento: una visión inclusiva de las diferentes variedades y registros de la lengua de instrucción, para garantizar la equidad lingüística. Ahora bien, la problemática se plantea en cómo combatir la desigualdad lingüística y las diferentes percepciones de sus hablantes.

De igual modo, la inclusión de la diversidad de variedades, niveles y registros en la lengua de instrucción facilita el proceso de aprendizaje lingüístico, dado que

$5 \quad$ En el original: "The academic and linguistic success of Spanish-dominant children in dual language programs can be attributed to the use of the curricular mainstream taught in the child's native language as well as rigorous content standards regardless of the language of instruction". 
una aproximación inclusiva, en positivo y no punitiva, rebaja los filtros afectivos del alumno (Krashen, 1985). Basándose en la quinta hipótesis sobre adquisición de un segundo idioma de Krashen en referencia al rol del filtro afectivo (Schultz, 2007) en el aprendizaje de un segundo idioma, Delpit ofrece dos recomendaciones: abrazar la diversidad lingüística en el aula apreciando sus múltiples expresiones y diseñar constantemente actividades que promuevan un pluralismo lingüístico. Según Delpit, "el llamado filtro afectivo es susceptible de aumentar cuando el aprendiz está expuesto a una corrección constante" 6 (2009, p. 92). En este sentido, rebajar el filtro afectivo, así como una exposición exhaustiva a contenidos de calidad, mejora el aprendizaje. Para abrazar la diversidad lingüística de los alumnos hispanohablantes, se debe buscar el difícil equilibrio entre corrección excesiva en la producción oral y escrita, y la oportuna aplicación de la norma estándar. El maestro de contenidos debe mostrar una actitud receptiva y abierta a la diversidad lingüística y cultural (Kubota, 2004), valorando la riqueza inherente de la diversidad de cualquier lengua. Para ello, se debe ofrecer la explicación normativa del lenguaje en caso de expresiones no normativas o erróneas, evitando las valoraciones punitivas de las diferentes variedades y dialectos.

Los programas de inmersión dual en California utilizan la lengua de instrucción en los contenidos de las clases de ciencias sociales o historia, ciencias naturales, tecnología, matemáticas, o educación física, por citar algunos ejemplos (Senesac, 2002; Potows$\mathrm{ki}, 2007)$. En estas aulas de aprendizaje integrado de contenidos, la lengua es el instrumento primordial para transmitir no solo el currículum, sino una percepción social y cultural de la lengua que influye en la construcción de las identidades lingüísticas y culturales de cada individuo. Este complejo proceso no se ve afectado solo por aspectos puramente lingüísticos, como las variedades, registros y diferentes

6 En el original: "The so-called affective filter is likely to be raised when the learner is exposed to constant correction". dialectos de una misma lengua. Las percepciones culturales, políticas y económicas se ven igualmente mediadas por la pertenencia a determinados grupos lingüísticos de sus hablantes, ya sean minoritarios o mayoritarios. Los programas de inmersión dual se han mostrado efectivos en equiparar los beneficios educativos de los latinos como minoría lingüística (Lindholm-Leary y Block, 2010). Por el contrario, la ausencia de una visión inclusiva que no considere el estatus social de esta minoría podría limitar la equiparación de sus beneficios (De Jong y Howard, 2009) y fomentar una triple segregación lingüística, económica y racial (Gándara, 2010). Un enfoque inclusivo en la instrucción del lenguaje, partiendo de la aceptación de esta multiplicidad de factores, resulta más enriquecedor, útil y fiel a una realidad multilingüe.

La clase de contenido en español, a la vez que ofrece un alto nivel en el currículum estándar, supone un enorme refuerzo no solo en la literacidad, sino en la comprensión de cuestiones metalingüísticas. En la clase de historia, por ejemplo, la discusión sobre la historicidad léxica o gramatical ayuda tanto al enriquecimiento cultural como a la mejor comprensión de la evolución del idioma. En este sentido, la clase de contenido apoya el refuerzo positivo de las variedades lingüísticas, ya que ofrece una reflexión sobre los mecanismos que han dado forma a esta lengua y a sus distintas variedades y dialectos. Trabajar en el análisis de textos históricos que representan diferentes registros lingüísticos y literarios (poesía, artículos periodísticos, cartas personales, etc.) ayudará a ilustrar las diferentes voces de sus narradores y protagonistas. De forma similar, en la clase de educación física, las palabras "alberca" o "zacate" han de ser igualmente bienvenidas tanto como sus sinónimos "piscina" o "hierba", ya que se valora la riqueza de las variedades de un idioma. El diseño de actividades para el aula de doble inmersión debe procurar incluir todas las variedades y registros del idioma. De esta manera, a la vez que se trabaja el contenido académico de la clase, se crean 
ocasiones de debate sobre el origen y las causas de estas distintas variedades. En la clase de tecnología, por ejemplo, un texto sobre "la desaparición de los cederrones en los ordenadores" puede precipitar una enriquecedora discusión sobre la evolución historicista del lenguaje y la influencia de las lenguas en contacto por proximidad geográfica, económica o política. El término "ordenador" es usado en España por influencia del francés ordinateur, a diferencia del uso de su sinónimo "computadora" en toda América, por influencia del inglés computer. En esta misma actividad se podría introducir además el concepto de neologismo para ilustrar cómo una lengua viva se transforma y evoluciona, como ocurre con la palabra "cederrón", derivada del inglés CD-ROM. Este proceso requiere del docente de inmersión dual un arduo trabajo de búsqueda de contenidos diversos y variedad lingüística y de significado para diseñar la instrucción. Asimismo, requiere un diseño de actividades que permitan el análisis progresivo o en múltiples capas (RodríguezValls, Solsona-Puig y Capdevila-Gutiérrez, 2017), donde la dificultad de la lengua y del contenido se adapten a la diversidad del alumnado.

El equilibro necesario que debe existir entre la instrucción del contenido y la de los aspectos lingüísticos, como han señalado Cammarata y Tedick (2012), es un reto añadido en las clases de inmersión. Según estos autores, un aspecto que podría influir en este reto es la constatación de que los de docentes CLIL no siempre poseen una sólida formación pedagógica en cuestiones de enseñanza del lenguaje. Una aproximación inclusiva a la diversidad del lenguaje podría equilibrar esta posible carencia, exponiendo a los alumnos de inmersión a un amplio abanico de fuentes ricas en matices lingüísticos. El estudio del discurso lingüístico, asociado a cada variedad o registro, sobre todo de una lengua global como el español (Paffey, 2014), debe ser un foco de interés en las aulas de inmersión dual en secundaria. Además, la inclusividad favorecerá una construcción más positiva de la representación social e identitaria propia a través de la diversidad lingüística del alumno hispanohablante (Rojas, 2014). Ello ayudará a que una variedad deje de ser percibida con más autoridad, elegancia o academicismo que otra. Por proximidad, y dado que el uso del inglés cubre un radio de acción más amplio que el español (Potowski, 2004), un enfoque inclusivo podría mitigar una situación similar en el aula.

\section{Repercusiones identitarias y transnacionales: inclusión y revalorización de las variedades lingüísticas en otros idiomas, programas y contextos educativos y sociales}

El dilema de incluir equitativamente las diferentes variedades de un mismo idioma de instrucción no solo afecta a los programas de inmersión dual en español. Ya sean mayoría o minoría en su contexto sociocultural, cualquier programa de inmersión en el que la lengua meta sea usada por un cuerpo de hablantes distribuidos en múltiples comunidades lingüísticamente diferenciadas puede verse afectado por aspectos no solo lingüísticos, sino también sociopolíticos. Como hablantes, los maestros y alumnos a portan al aula sus diversas variedades del idioma, no exentas de prejuicios y estigmas socioeconómicos. Un ejemplo de este aspecto lo aporta el estudio sociolingüístico de Starr sobre la influencia de las variedades de mandarín utilizadas por los maestros en un programa de inmersión en la costa oeste de la EE.UU. Este estudio señala que el uso de una de las variedades no estándar aparecía "consistentemente estigmatizado como un signo de bajo nivel de educación en las comunidades de hablantes de mandarín"7 (2010, p. 125). A pesar de ello, el estudio concluye que su uso es valioso e inseparable de la identidad del maestro, y que la exposición a múltiples variedades redunda en un mejor conocimiento de las variantes de la lengua de inmersión $y$, por ende, del idioma en conjunto. Otro estudio, de

7 En el original: "Consistently stigmatized as a sign of low education in Mandarin-speaking communities". 
Lasagabaster y Huguet (2007), analiza las actitudes lingüísticas de más de 1.800 futuros maestros en nueve comunidades bilingües en Europa con cuestionarios en nueve idiomas (vasco, catalán, holandés, francés, inglés, flamenco, frisón, irlandés y español). Según estos investigadores, las percepciones que los hablantes bilingües tienen de sus lenguas minoritarias, mayoritarias o terceras lenguas, generalmente conocidas como L3 o extranjeras, dependen en gran medida de su contexto social. En la formación de estas actitudes lingüísticas en entornos multilingües, en cuya construcción la familia, la sociedad y la escuela juegan un rol primordial, la influencia de los docentes es extremadamente importante y duradera en el tiempo. Sin filtro o enmienda, dichas percepciones sesgadas podrían llegar a infiltrarse en las aulas a través de los futuros docentes. El caso de España es especialmente llamativo, ya que, según Huguet, Lasagabaster y Vila (2008), más de un 40\% de la población vive en áreas bilingües donde el lenguaje minoritario puede no ser oficial ni ser, por tanto, parte del sistema educativo. En el citado artículo también se asevera que, alli donde se han hecho esfuerzos para implementar programas bilingües, los resultados confirman la hipótesis de Cummins sobre la interdependencia en las transferencias lingüísticas (1979).

En algunos contextos multilingües, como Ouebec y Cataluña, donde existe una larga tradición de programas de inmersión en la lengua de la llamada mayoría lingüística frágil (McAndrew, 2013), los estudiantes de inmersión en francés y catalán conviven en un entorno nacional dominado por el inglés y el español, respectivamente. Incluso en estos contextos, el debate sobre los aspectos socioeconómicos, el papel de la inmigración y la construcción de la identidad han tenido una constante y, con frecuencia, controvertida actualidad. Tarone y Swain (1995) intentaron responder al interrogante planteado en las aulas de inmersión en francés de Canadá de por qué los estudiantes dejaban progresivamente de usar la lengua meta entre iguales, a medida que progresaban en la educación primaria. La respuesta podría estar, según estos autores, en que el uso casi exclusivo del lenguaje académico en clase de ese segundo idioma se aleja del registro utilizado por los adolescentes en la construcción de su identidad. La elección de este registro no académico depende de factores socioeconómicos y les sirve a estos jóvenes para afirmar su identidad. En otro estudio sobre una escuela de inmersión en catalán, Vila Moreno (1996) llegó a una conclusión similar: el uso de la lengua de instrucción para usos no académicos depende de factores de índole más social que estrictamente académica. Un enfoque inclusivo en la instrucción debe servir también para que los hablantes nativos mejoren las actitudes lingüísticas hacia su lengua nativa, sea cual sea su variante o registro, al tiempo que los hablantes de un segundo idioma puedan integrar plenamente todos sus posibles registros. Según Rojas (2014), esto supone un aspecto clave en la construcción de la representación social e identitaria propia. Cummins y Early (2010) usaron una metodología de estudio de casos para investigar 18 ejemplos en múltiples países sobre la creación de textos y la construcción de la identidad en grupos lingüísticos minoritarios. Como resultado de un estudio previo sobre la integración de lenguas y contenidos, surgió la idea de los textos identitarios como una estrategia para incrementar la equidad educativa en grupos más susceptibles de ser marginados por su origen lingüístico, económico o social: "La creación de textos identitarios es un componente de una aproximación pedagógica más amplia que articula un discurso alternativo a la devaluación implícita de las habilidades, lenguas, culturas e identidades de los estudiantes que ocurre en las aulas, donde las vías preferentes para crear significado y los lenguajes nativos son ignorados o tratados con una 'negligencia benévola'”8 (Cummins y Early, 2010, p. 4).

8 En el original: "Identity text creation is one component of a broader pedagogical approach that articulates a 'counterdiscourse' to the implicit devaluation of students' abilities, languages, cultures, and identities that occurs in classrooms where students' preferred ways of meaning making and home languages are ignored or treated with 'benign neglect"'. 
Palmer (2007) utiliza un concepto similar, al que denomina "racismo benévolo", en su estudio etnográfico realizado en una escuela donde el programa de inmersión dual era minoritario. Como es frecuente, muchos de estos programas conforman una rama (strand) en un contexto educativo predominantemente anglófono. En su estudio advierte que tratar de forma condescendiente, sin ofrecer un discurso alternativo para contrarrestar la cultura dominante, puede socavar la habilidad de los estudiantes de minorías para construir una imagen positiva de sus identidades, lo que afectaría su rendimiento académico. En sus conclusiones, Cummins y Early (2010) apuntan que el peso de una instrucción equitativa y respetuosa con la multiplicidad de lenguas recae esencialmente en el maestro. Esta aseveración también sería cierta dentro de un aula con múltiples variedades de una misma lengua. El docente requiere, como veremos a continuación, de una formación que lo prepare para poder gestionar un aula inclusiva, siendo a la vez conocedor de las múltiples variedades, registros y culturas asociadas a su lengua de instrucción.

\section{Preparación de maestros para la enseñanza del español de manera inclusiva}

En las secciones anteriores se ha analizado la voluntad de crear un aula inclusiva, ya sea durante la instrucción de lengua o cuando se enseñan otros contenidos. En ambos casos, el rol del maestro como facilitador y promotor de todos los registros que se manejan en el aula es clave. El maestro que promueve la inclusión lingüística debe ser un maestro que entienda el español en su doble naturaleza: lengua como recurso y lengua como identidad sociopolítica (Planas y Civil, 2013). En este apartado, en el que discutimos la lengua como identidad sociopolítica, la tipología del español se define por su carácter cambiante y su movilidad. A medida que el español se mueve desde la frontera con México hacia el norte empieza a perder ese carácter de lengua de dos espacios geopolíticos y se adapta a la tipología socioeconómica de cada localidad. Por ejemplo, el español de Los Ángeles es un idioma que representa un estatus social determinado, una clase trabajadora que amalgama hablantes de diferentes países (México, El Salvador, Nicaragua, Guatemala...). Al respecto, Alonso, Durand y Gutiérrez explican que el predominio del uso del español "tiene que ver con orígenes de clase o región de origen [...], entre los trabajadores agrícolas, por ejemplo, solo se habla en español" (2008, p. 18).

El español de la metrópolis y sus suburbios es un español que vive en la calle y en el mercado, y es en estos espacios donde la lengua florece en todas sus variedades y acepciones. Esta tendencia persiste, si seguimos más al norte, ya que en el valle central de California el español es la lengua de interacción en el campo. Allí, como en los otros espacios, los estudiantes viven, aprenden y usan el español que oyen de sus padres, en la radio y la televisión. Para complementar este aprendizaje del español en casa, el maestro tiene que estar capacitado para expandir, aumentar y analizar el carácter mutante, la estratificación y la movilidad del mismo.

En la actualidad, la capacitación y preparación de maestros bilingües en California se limita, en la mayoría de los casos, a dos cursos. En ellos, los candidatos a maestros, aprenden las metodologías que se pueden usar en las aulas de doble inmersión, así como los aspectos culturales que enmarcan una educación inclusiva. Además de estos dos cursos, los candidatos deben completar horas de práctica en una escuela de doble inmersión. Aunque estos requisitos preparan de algún modo a los candidatos, creemos necesario que los programas de preparación añadan experiencias que faciliten el análisis de las diferentes variedades del español. A tal efecto, proponemos dos tipos de actividades formativas: por un lado, un trabajo etnográfico en diferentes comunidades donde se habla español (study away) dentro del mismo estado donde se va a enseñar; por otro lado, el intercambio de maestros con otros países de habla hispana (study abroad). 
La primera actividad formativa de trabajo etnográfico potenciaría un mejor conocimiento de la población a la que el futuro maestro tendrá acceso. El candidato a maestro bilingüe debería visitar diferentes comunidades de habla hispana y analizar las diferentes variedades de español y los modismos peculiares de la zona. Esta investigación ayudará al candidato a ver cómo, cuándo y por qué la comunidad utiliza el español. Tal y como explica Velasco Orozco (2003), la investigación etnográfica "se interesa por lo que la gente hace, cómo se comporta, cómo interactúa. Se propone descubrir sus creencias, valores, perspectivas, motivaciones y el modo en que todo eso se desarrolla" (p. 159). Más aún, este análisis epistemológico de la lengua, definida esta como una reflexión omnímoda y creativa, ayudará al maestro a entender, como explica Gallardo en su análisis de la obra de Wittgenstein, "los procesos mentales que acompañan a las expresiones en que se utilizan las palabras" (2011, p. 89).

La segunda actividad, de intercambio internacional y visita a otro país de habla hispana, tendría un doble objetivo. Por un lado, facilitaría la familiarización con diversos grupos y culturas hispanohablantes, a la vez que la exposición a diversos registros lingüísticos. Por otro lado, supondría una inmersión en el ámbito educativo, lo cual favorece la exposición del docente al español académico. Esto último sería particularmente eficaz, ya que, con frecuencia, los futuros docentes, pese a ser bilingües, carecen del vocabulario específico y académico, debido a su formación esencialmente en inglés.

La inclusividad lingüística no debería, en ningún caso, generar la existencia de un estudiante pseudobilingüe, altamente competente en un idioma, pero con destrezas lingüísticas limitadas en el otro. Tal y como se expuso en la introducción, las lenguas crean identidades que definen a las personas. Una aproximación inclusiva a la enseñanza del español debe resultar en un estudiante con una identidad políglota, cosmopolita y global. Sin esta identidad se corre el peligro, como explica Castells, de que el es- tudiante se sienta oprimido por el sistema, y añade: "cuando se trata de identidad, los contextos social, cultural y político son decisivos. Considero que tengo algo que decir al respecto, no solo teórica sino también personalmente, en tanto que me siento catalán, una identidad que ha sido oprimida en los últimos 500 años"9 (2008, p. 5).

Los procesos y requisitos para la formación de profesorado bilingüe deben incluir contenidos, estrategias y prácticas que doten al maestro de una visión plural de la lengua de instrucción y sus múltiples culturas. Solo así el maestro del aula de inmersión dual será capaz de llegar a la excelencia en la enseñanza, bien sea de lengua o de contenidos, a la vez que ayudará a crear en el alumno un sentimiento identitario positivo por su lengua y su cultura.

\section{Conclusiones}

Este artículo presenta un análisis preliminar del concepto de inclusividad lingüística y de la pluralidad de las múltiples variedades y registros de una lengua de instrucción en un entorno multilingüe, así como sus repercusiones tanto en la construcción de la identidad de los hablantes de lenguas minoritarias y como en la capacitación docente. A partir de este análisis, el artículo arroja diversas conclusiones. En primer lugar, surge la premisa de que el docente debe promover un aprendizaje riguroso con una actitud de inclusividad lingüística, para favorecer el aprendizaje, al tiempo que respeta la diversidad lingüística y cultural. En segundo lugar, se deben valorar las diferencias y reforzar las similitudes de las distintas variedades para asegurar una sólida base lingüística, así como una construcción identitaria y cultural equilibrada del alumnado. En tercer lugar, es necesario enfatizar la utilización de la clase de lengua y la de contenido en español

9 En el original: "When it comes to identity, the cultural context, the social context and the political context are decisive. I think I can say something not only about the theoretical but about the personal too, about feeling myself Catalan, which has for the last 500 years or so been an oppressed identity". 
con un objetivo triple: como plataforma para una educación de excelencia, como refuerzo lingüístico en la lengua meta, como ejemplo de inclusividad y respeto de la variedad lingüística y cultural del aula. Asimismo, y sin perder de vista el contexto - en este caso, el del español en las aulas de inmersión dual en California-, se requiere la combinación de todos estos elementos para contribuir a diluir los prejuicios culturales y socioeconómicos asociados al uso de esta lengua de instrucción. Con todo ello se consigue avanzar hacia la construcción de un modelo de instrucción de calidad, transferible a la enseñanza en cualquier otro idioma.

Una comparación de determinados aspectos de los programas de inmersión en español en California con otras lenguas de inmersión y otros países multilingües ha permitido establecer los siguientes paralelismos generalizables. En primer lugar, el uso de la lengua de instrucción minoritaria dentro y fuera de los contextos académicos se supedita a los usos lingüísticos socialmente mayoritarios. En segundo lugar, las condiciones sociolingüísticas de los hablantes de minorías lingüísticas afectan la percepción de sí mismos y el proceso de construcción de su identidad. Por último, en el maestro recae la responsabilidad última de transmitir una percepción equitativa y lingüísticamente inclusiva con las variedades y niveles de lengua.

Finalmente, en referencia a los programas de capacitación docente, el presente artículo propone la incorporación de dos actividades de carácter et- nográfico y de intercambio cultural para garantizar el necesario enfoque multicultural del docente en las aulas de inmersión dual y mejorar su preparación y conocimiento de la lengua y cultura meta. Así mismo, se recomienda que futuros estudios analicen cómo los programas de preparación de maestros evalúan las destrezas de sus candidatos en las áreas propuestas en este artículo. Sin este tipo de evaluaciones, se corre el riesgo de preparar nuevas generaciones de maestros que, aunque tengan conocimiento del concepto de inclusión lingüística, carezcan de la capacidad de "revelar oportunidades para la esperanza, sin importar cuales sean los obstáculos"10 (Freire, 2014, p. 4). Ciertamente, la inclusividad lingüística no es una tarea sencilla. Bien sea como formadores de maestros, como administradores o como docentes de programas de inmersión dual, se debería enfatizar su aplicación como paradigma de equidad sociolingüística. Una sociedad que solo reconoce y apoya la supremacía de una lengua, una voz o un registro determinado sobre otros nunca podrá considerarse democrática, participativa e inclusiva. Los programas de inmersión dual deben contribuir y formar parte de ese esperanzador cambio hacia una sociedad que se esfuerza por equilibrar la balanza de la desigualdad. En definitiva, proponemos que la inclusividad lingüística sea una premisa sine qua non para las futuras investigaciones sobre instrucción en entornos multilingües, capacitación de maestros, su afectación en las actitudes lingüísticas y la propia construcción de la identidad a través del lenguaje.

10 En el original: "Unveil opportunities for hope, no matter what the obstacles may be". 


\section{Bibliografía}

Ada, A. F.y Zubizarreta, G. M. (2013). Nacer bailando. Nueva York: Atheneum.

Alanís, I., y Rodríguez, M. A. (2008). Sustaining a dual language immersion program: Features of success. Journal of Latinos and Education, 7(4), 305-319. doi: 10.1080/15348430802143378

Alba, O. (2001). El español estándar desde la perspectiva dominicana. Brigham Young University. All Faculty Publications. 1087. Recuperado de https://scholarsarchive.byu.edu/facpub/1087-

Alfonso, T. (2017). Programas de doble inmersión: educación bilingüe y lenguas minoritarias en Estados Unidos y México. Pedagogía y Saberes, (47), 107-119. doi:10.17227/01212494.47pys107.119

Alim, S. H. (2005). Critical language awareness in the United States: Revisiting issues and revisiting pedagogies in a resegregated society. Educational Researcher, 34(7), 24-31. Recuperado de https://www.jstor.org/stable/3699797

Alonso, J. A., Durand, J. y Gutiérrez, R. (2008). La persistencia del español en los colectivos hispanos de Estados Unidos: Una introducción. En J. A. Alonso, J. Durand y R. Gutiérrez (eds.), El futuro del español en los Estados Unidos: La lengua en las comunidades de migrantes hispanos (pp.1-46). Madrid: Ariel.

Altbach, P. G. (2007). The International Imperative in Higher Education. Róterdam, Holanda: Sense Publishers.

Anzaldúa, G. (2012). Borderlands/La frontera: The new Mestiza. San Francisco: Aunt Lute Books.

Betancur-García, M. C. (2014). Mestizaje lingüístico y cultural. Revista Venezolana de Análisis de Coyuntura, 20(2), 103-129. Recuperdado de http://www.redaly c.org/pdf/364/36440846006.pdf

CAL - Center for Applied Linguistics (2018a). Growth of the TWI programs, 1962-present. Recuperado de http:// www.cal.org/twi/directory/twigrow.htm

CAL - Center for Applied Linguistics (2018b). Language of Initial Literacy Instruction in Two-Way Immersion Programs. Recuperado de http://www.cal.org/twi/literacylanguage.htm

Cammarata, L., y Tedick, D. J. (2012). Balancing content and language in instruction: The experience of immersion teachers. The Modern Language Journal, 96(2), 251-269. doi:10.1111/j.1540-4781.2012.01330.x

Cassany, D. (2011). Prácticas lectoras democratizadoras. Textos de Didáctica de la Lengua y la Literatura, (58): 29-40.

Castells, M. (2008). Globalization identity and the state. Social Dynamics: A Journal of African Studies, 26(1), 5-17. doi: $10.1080 / 02533950008458682$

CDE - California Department of Education (2018a). Facts about English Learners in California. Sacramento. California Department of Education. Recuperado de https://www.cde.ca.gov/ds/sd/cb/cefelfacts.asp

CDE - California Department of Education (2018b). Two-Way Language Immersion Program FAQ. Sacramento. California Department of Education. Recuperado de https://www.cde.ca.gov/sp/el/ip/faq.asp

CLI - California Legislative Information (2018). Senate Bill 1174. Relating to English language education. Recuperado de https://leginfo.legislature.ca.gov/faces/billNavClient.xhtml?bill_id=201320140SB1174 
ISSN 0123-1294 | e-ISSN 2027-5358 | Educ.Educ. Vol. 21. No. 2 | Mayo-agosto de 2018 | pp. 219-236.

Universidad de La Sabana | Facultad de Educación

Cummins, J. (1979). Linguistic interdependence and the educational development of bilingual children. Review of Educational Research, 49, 222-251. doi:10.2307/1169960

Cummins, J. y Early, M. (2010). Identity Texts: The Collaborative Creation of Power in Multilingual Schools. Stokeon-Trent, Reino Unido: Trentham Books.

De Jong, E. J. (2016). Two-way immersion for the next generation: Models, policies, and principles. International Multilingual Research Journal, 10(1), 6-16. doi:10.1080/19313152.2016.1118667

De Jong, E. J. y Howard, E. (2009). Integration in two-way immersion education: Equalising linguistic benefits for all students. International Journal of Bilingual Education and Bilingualism, 12(1), 81-99. doi: $10.1080 / 13670050802149531$

Delpit, L. (2009). Language diversity and learning. En VV.AA., The critical pedagogy reader (pp. 324-337). Nueva York: Rutledge.

Fernández-Vítores, D. (2017). El Español, una lengua viva. Informe 2017. Madrid: Instituto Cervantes. Recuperado de https://cvc.cervantes.es/lengua/espanol_lengua_viva/pdf/espanol_lengua_viva_2017.pdf

Freire, P. (2014). Pedagogy of hope: Reliving pedagogy of oppressed. Nueva York: Bloomsbury Academic.

Gallardo, A. (2011). Wittgenstein: Epistemología y lenguaje. Praxis, 66, 87-97. Recuperado de http://www.revistas. una.ac.cr/index.php/praxis/article/view/3976

Gándara, P. (2010). Overcoming Triple Segregation. Educational Leadership, 68(3), 60-64.

García, O. y Kleifgen, J. A. (2010). Educating emergent bilinguals: Policies, programs and practices for English Language Learners. Nueva York: Teachers College Press.

Ghosh, R., y Galczynski, M. (2014). Redefining multicultural education: Inclusion and the right to be different. Toronto: Canadian Scholars' Press.

Hornberger, N. H. (2009). La educación multilingüe, política y práctica: Diez certezas. Revista Guatemalteca de Educación, 1(1), 1-44.

Howard, E. R., Lindholm-Leary, K. J., Sugarman, J., Christian, D. y Rogers, D. (2007). Guiding principles for dual language education. Washington: Center for Applied Linguistics. Recuperado de www.cal.org/twi/pdfs/ guiding-principles.pdf

Huguet, Á., Lasagabaster, D. y Vila, I. (2008). Bilingual education in Spain: present realities and future challenges. En Encyclopedia of language and education (pp.1672-1682). EE.UU.: Springer.

Ibarra, J. (2012). La integración lingüística del alumnado inmigrado. Textos de didáctica de la lengua y la literatura, 61, 9-21.

Krashen, S. D. (1985). The input hypothesis: Issues and implications. Toronto: Addison-Wesley Longman.

Kubota, R. (2004). Critical multiculturalism and second language education. En B. Norton (ed.), Critical pedagogies and language learning (cap. 3). Vancouver: University of British Columbia.

Lasagabaster, D. y Huguet, A. (eds.) (2007). Multilingualism in European bilingual contexts: Language use and attitudes (vol. 135). Clevedon, Reino Unido: Multilingual Matters. 
Lasagabaster, D. y Sierra, J. M. (2009). Immersion and CLIL in English: more differences than similarities. ELT journal, 64(4), 367-375. doi:10.1093/elt/ccpo82

Lindholm-Leary, K. J. (2005). The rich promise of two-way immersion. Educational Leadership, 62(4), 56-59.

Lindholm-Leary, K. J. y Block, N. (2010). Achievement in predominantly low SES/Hispanic dual language schools. International Journal of Bilingual Education and Bilingualism, 13(1), 43-60. doi:10.1080/13670050902777546

Lindholm-Leary, K. y Hernández, A. (2011). Achievement and language proficiency of Latino students in dual language programmes: Native English speakers, fluent English/previous ELLs, and current ELLs. Journal of Multilingual and Multicultural Development, 32(6), 531-545. doi: 10.1080/01434632.2011.611596

Livingstone, K. A. y Ferreira, A. (2009). La efectividad de un modelo metodológico mixto para la enseñanzaaprendizaje de español como lengua extranjera. Boletín de Filología, 44(2), 89-118. doi: 10.4067/So71893032009000100004

Lomas, C. (2014). La educación lingüística, entre el deseo y la realidad. En C. Lomas (ed.), La educación lingüística, entre el deseo y la realidad: competencias comunicativas y enseñanza del lenguaje (pp. 9-17). México: Flacso.

Marian, V., Shook, A. y Schroeder, S. R. (2013). Bilingual two-way immersion programs benefit academic achievement. Bilingual Research Journal, 36(2), 167-186. doi:10.1080/15235882.2013.818075

Martín-Peris, E. (2010). ¿A qué nos referimos cuando hablamos de "usar una lengua para aprenderla"? Bellaterra Journal of Teaching and Learning Language and Literature, 3(1), 1-18. doi:10.5565/rev/jtl3.210

McAndrew, M. (2013). Fragile Majorities and Education: Belgium, Catalonia, Northern Ireland, and Quebec. Kingston: McGill-Queen's Press-MQUP.

Montrul, S. (2012). El bilingüismo en el mundo hispanohablante. Malden: John Wiley \& Sons.

Nero, S.y Ahmad, N. (2014). Vernaculars in the classroom: Paradoxes, pedagogy, possibilities. Nueva York: Routledge.

Paffey, D. (2014). Language ideologies and the globalization of "standard"Spanish. Broadway: Bloomsbury Academic.

Palmer, D. (2007). A dual immersion strand programme in California: Carrying out the promise of dual language education in an English-dominant context. International Journal of Bilingual Education and Bilingualism, 10(6), 752-768. doi: 10.2167/beb397.0

Planas, N. y Civil, M. (2013). Language-as-resource and language-as-political: Tensions in the bilingual Mathematics classroom. Mathematics Education Research Journal, 25(3), 361-378. doi: 10.1007/s13394-013-0075-6

Potowski, K. (2004). Student Spanish use and investment in a dual immersion classroom: Implications for second language acquisition and heritage language maintenance. The Modern Language Journal, 88(1), 75-101. doi: 10.1111/j.0026-7902.2004.00219.x

Potowski, K. (2007). Language and identity in a dual immersion school (vol. 63). Clevedon, Reino Unido: Multilingual Matters. 
Rodríguez, D., Carrasquillo, A. y Soon-Lee, K. (2014). The bilingual advantage: Promoting academic development, biliteracy and native language in the classroom. Nueva York: Teachers College Press.

Rodríguez-Valls, F. (2009). Culturally relevant poetry: Creating Esperanza (hope) with stanzas. Multicultural Education, $17(1), 10-13$.

Rodríguez-Valls, F.y Capdevila-Gutiérrez, M. (2017). ¿Qué voces escuchas? Aproximación a la lectura de cuentos a través de los ojos de los héroes silenciosos. Bilingual Review/Revista Bilingüe, 33(4), 63-75. Recuperado de http://bilingualreview.utsa.edu/index.php/br/article/view/176/119

Rodríguez-Valls, F., Solsona-Puig, J. y Capdevila-Gutiérrez, M. (2017). Teaching social studies in Spanish in dual immersion middle schools: A biliterate approach to history. Cogent Education, 4(1). doi: $10.1080 / 2331186 X .2017 .1326202$

Rojas, D. (2014). Estatus, solidaridady representación social de las variedades dela lengua española entre hispanohablantes de Santiago de Chile. Literatura y lingüistica, 29, 251-270. doi:10.4067/S0716-58112014000100014

Schütz, R. (2007). Stephen Krashen's theory of second language acquisition. English made in Brazil, 2(2), 2007.

Senesac, B.V. K. (2002). Two-way bilingual immersion: A portrait of quality schooling. Bilingual Research Journal, 26(1), 85-101. doi: 10.1080/15235882.2002.10668700

Shin, S. J. (2012). Bilingualism in schools and society: Language, identity, and policy. Nueva York: Routledge.

Showstack, E. (2012). Symbolic power in the heritage language classroom: How Spanish heritage speakers sustain and resist hegemonic discourses on languages and cultural diversity. Spanish in Context, 9(1), 1-26. doi: 10.1075/sic.9.1.01sho

Starr, R. L. (2010). Teaching the Standard without Speaking the Standard:Variation Among Mandarin-Speaking Teachers in a Dual-Immersion School. University of Pennsylvania Working Papers in Linguistics, 15(2). Recuperado de https://repository.upenn.edu/cgi/viewcontent.cgi?article=1136\&context=pwpl

Tarone, E. y Swain, M. (1995). A sociolinguistic perspective on second language use in immersion classrooms. The Modern Language Journal, 79(2), 166-178. doi: 10.2307/329617

Trujillo-Sáez, F., Lorenzo, F. y Vez, J. M. (2011). Educación bilingüe. Integración de contenidos y segundas lenguas. Madrid: Sintesis.

USCB - United States Census Bureau (2018a). California Quick Facts. Recuperado de https://www.census.gov/ quickfacts/fact/table/CA,US/PSTO45217

USCB - United States Census Bureau (2018b). Language spoken at home: California. Recuperado de https://factfinder.census.gov/faces/tableservices/jsf/pages/productview.xhtml?pid=ACS_16_5YR_ S1601\&prodType=table

Velasco-Orozco, J. J. (2003). La investigación etnográfica y el maestro. Tiempo de Educar, 4(7), 153-169. Recuperado de http://www.redaly c.org/pdf/311/31100706.pdf

Vila-Moreno, F.X. (1996). When classes are over. Language choice and language contact in bilingual education in Catalonia. Disertación Doctoral. Bruselas: Vrije Universiteit Brussel. 